\title{
Détournements savants du jazz en France et en Allemagne (1919-1922) : Adieu New-York ! de Georges Auric et le " Ragtime " de la Suite 1922 de Paul Hindemith Hijacking Jazz in France and Germany (1919-1922): Georges Auric's Adieu New-York! and Paul Hindemith's "Ragtime” from Suite 1922
}

\section{Martin Guerpin}

Volume 14, numéro 2, automne 2013

La passion de la recherche (à la mémoire de Maryvonne Kendergian)

URI : https://id.erudit.org/iderudit/1023741ar

DOI : https://doi.org/10.7202/1023741ar

Aller au sommaire du numéro

\section{Éditeur(s)}

Société québécoise de recherche en musique

ISSN

1480-1132 (imprimé)

1929-7394 (numérique)

Découvrir la revue

Citer cet article

Guerpin, M. (2013). Détournements savants du jazz en France et en Allemagne (1919-1922) : Adieu New-York ! de Georges Auric et le « Ragtime » de la Suite 1922 de Paul Hindemith. Les Cahiers de la Société québécoise de recherche en musique, 14(2), 69-77. https://doi.org/10.7202/1023741ar

\section{Résumé de l'article}

La plupart des travaux consacrés à l'influence du jazz dans le domaine de la musique savante s'attachent à relever les points communs et les différences de certaines oeuvres avec le modèle musical auquel elles empruntent.

À partir de deux oeuvres représentatives de ces emprunts au début des années 1920, Adieu New-York ! de Georges Auric (1919) et le « Ragtime » de la Suite 1922 de Paul Hindemith, cet article propose d'élargir la perspective comparatiste à des problématiques relevant du domaine des transferts culturels, afin de dégager la dimension nationale de la réception et de l'utilisation du jazz dans la musique savante au début des années 1920, en France et en Allemagne.

De quels répertoires de jazz Auric et Hindemith ont-ils pu avoir connaissance ? Comment caractériser la manière particulière dont leurs oeuvres empruntent à ces répertoires ? En quoi des spécificités nationales jouent-elles un rôle dans la manière dont les compositeurs savants utilisent le jazz dans leurs oeuvres ? Loin de chercher à reproduire fidèlement ces musiques, Adieu New-York! et le " Ragtime " de la Suite 1922 détournent les caractéristiques musicales du jazz afin de proposer de nouvelles voies à la musique savante, hors des canons esthétiques issus du romantisme. Le jazz apparaît ainsi comme un moyen pour de jeunes compositeurs d'affirmer leur singularité. Dans le même temps, chacune de ces oeuvres emprunte paradoxalement à une musique alors clairement identifiée comme étrangère pour affirmer sa propre identité musicale nationale. Alors qu'Adieu New-York ! peut être considéré comme la mise en oeuvre de la redéfinition de l'identité musicale française proposée par Jean Cocteau dans LeCoq et l'Arlequin, le "Ragtime » de Paul Hindemith et son esthétique du laid s'inscrivent en droite ligne dans un courant expressionniste alors en vogue dans la jeune République de Weimar.
Tous droits réservés @ Société québécoise de recherche en musique, 2013
Ce document est protégé par la loi sur le droit d'auteur. L'utilisation des services d'Érudit (y compris la reproduction) est assujettie à sa politique d'utilisation que vous pouvez consulter en ligne.

https://apropos.erudit.org/fr/usagers/politique-dutilisation/ 


\section{Détournements savants du jazz en France et en Allemagne (1919-1922): Adieu New-York! de Georges Auric et le «Ragtime» de la Suite 1922 de Paul Hindemith}

\author{
Martin Guerpin \\ (Université Paris-Sorbonne/ \\ Université de Montréal)
}

$\mathrm{L}$

es emprunts jazz dans la musique savante européenne à la charnière des années 1920 ne constituent pas un sujet nouveau pour les musicologues (voir Hodeir 1954, Budds 2002, Perret 2003 et Mawer 2010¹). Fondateurs, ces travaux se caractérisent par une même approchequi consiste à mesurer la fidélité de ces emprunts aux modèles qui s'offraient alors aux compositeurs et à juger de la valeur des œuvres en fonction de cette fidélité. À propos du «Ragtime» de l'Histoire du soldat (1917) d'Igor Stravinski (1882-1971), le musicologue et compositeur français André Hodeir évoque ainsi un «contre-sens stupéfiant»:

Pour ne l'avoir pas compris, nos auteurs n'ont tiré du jazz qu'une infime partie de sa substance. Sur le plan esthétique comme sur le plan technique, les œuvres (ou fragments d'œuvres) que nous avons citées dans cette étude ne peuvent être regardées que comme de graves échecs² (Hodeir 1954, 236).

Ces lignes appellent deux remarques. Premièrement, le jazz qui sert de pierre de touche à la comparaison et au jugement de Hodeir est celui que l'historiographie traditionnelle de cette musique considère aujourd'hui comme le jazz des années 1920 : celui de Louis Armstrong, de Duke Ellington ou encore de Fletcher Henderson, pour ne prendre que quelques exemples connus ${ }^{3}$. Or, une étude des catalogues de partitions et de disques distribués en Europe au début des années 1920 permet de constater que le jazz entendu par les compositeurs français ou allemands au début des années 1920 était bien différent. Deuxièmement, plutôt que d'évaluer la fidélité des emprunts au jazz, en relevant des points communs et des différences, il apparaît plus productif de partir de l'hypothèse selon laquelle les incompréhensions dont fait mention Hodeir, loin de relever de l' 'échec», sont en réalité volontaires. Les déformations que les compositeurs font subir au jazz possèdent elles-mêmes un sens et peuvent par conséquent être analysées puis interprétées comme autant de prises de positions esthétiques ou, pour le dire autrement, comme des détournements savants du jazz.

Il s'agira donc de faire apparaitre les différentes manières dont les compositeurs opèrent ces détournements, mais aussi, et c'est là le point nodal de cet article, de montrer en quoi ces différences trouvent leur origine dans les préoccupations esthétiques propres à chaque compositeur. Une telle question en appelle une dernière: en quoi ces préoccupations sontelles elles-mêmes en partie conditionnées, d'une part, par des tendances musicales à l'œuvre dans les différents pays où le jazz a été utilisé dans la musique savante et, d'autre part, par les représentations qu'il véhiculait dans ces pays?

Afin de mettre en évidence les spécificités nationales des détournements du jazz dans la musique savante, une étude comparative de pièces recourant à cette musique en France et en Allemagne sera menée à partir de deux exemples: Adieu New-York! de Georges Auric (1899-1983), composé à Paris en 1919, et le «Ragtime» de la Suite 1922, du compositeur allemand Paul Hindemith (1895-1963). Le choix de ces deux œuvres réside à la fois dans leur caractère représentatif des détournements du jazz en France et en Allemagne au début des années 1920, et sur le fait que les études qui leur sont consacrées restent rares ${ }^{4}$.

1 D'autres travaux évoquent de manière plus générale la réception du jazz dans la musique savante française de l'entre-deux-guerres. Voir, entre autres, Gendron 2002, Ross 2007 et Guerpin dans Cerchari, Cugny et Kerschbaumer 2012.

2 C'est moi qui souligne. Les autres œuvres citées par André Hodeir sont la Création du Monde (1923) de Darius Milhaud (1892-1974) et le Concerto en sol (1929-1930) pour piano de Maurice Ravel (1875-1937).

3 Ébauchée dans les ouvrages du musicographe français Hugues Panassié dès les années 1930, cette historiographie traditionnelle s'institutionnalisa à partir de 1968 avec la parution d'Early Jazz, Its Roots and Musical Development de Gunther Schuller.

4 Évoquées dans quelques ouvrages traitant des rapports entre le jazz et la musique savante dans l'entre-deux-guerres (notamment Perloff 1991 et Budds 2002), ces deux œuvres n'ont jamais, à ma connaissance, fait l'objet d'analyses ou d'études qui leur soient exclusivement consacrées. 


\section{Adieu New-York! de Georges Auric ou Le Coq et l'Arlequin en musique}

Dégager la portée esthétique d'Adieu New-York! nécessite en premier lieu d'identifier le type de jazz dont Auric s'est inspiré, puis la manière dont il l'exploite.

\section{Un fox-trot déformé par une esthétique de la fausse note}

En 1918, au moment où Auric entreprend la composition d'Adieu New-York!' , le jazz peut être principalement entendu à Paris dans le cadre de revues données dans des music-halls ${ }^{6}$ et désigne un ensemble de genres musicaux plutôt qu'un type d'orchestration et une série de procédés musicaux. On identifie en effet le jazz au fox-trot, au blues ou au ragtime, plus ancien.

Outre ces revues, c'est par le biais de partitions que les compositeurs de la fin des années 1910 eurent accès au jazz ${ }^{7}$. Si Auric, dans sa correspondance et dans ses écrits, ne cite pas de source précise, il est un fait avéré qu'Érik Satie (1866-1925), dans le «Ragtime du Paquebot» de Parade (1917), fait référence à un ragtime intitulé That Mysterious $\operatorname{Rag}^{8}$ alors édité en France, et dont il a pu consulter la partition.

Le compositeur Jean Wiener (1896-1982), alors proche d'Auric, indique par ailleurs dans son autobiographie, Allegro Appassionato $(1978,92)$, que le jazz qu' il connaissait à la charnière des années 1920 était celui de la Tin Pan Alley ${ }^{9}$, disponible en France sous la forme de partitions de Ray Henderson (1896-1970), de Jerome Kern (1885-1945), ou encore d'Irving Berlin (1888-1989).

They Didn't Believe Me (figure 1), un fox-trot de Kern qui figure dans le catalogue de l'éditeur Salabert en 1914, constitue un parfait exemple de ce jazz sur partition auquel les compositeurs français ont pu avoir accès.

Ce fox-trot se caractérise par la «pompe» jouée par la main gauche du piano, qui fait alterner la basse sur la partie forte du temps et les notes principales de l'accord sur la partie faible; par un langage tonal fonctionnel dont le vocabulaire harmonique correspond à celui utilisé par les compositeurs de la première École de Vienne; par une
Figure 1: Jerome Kern, They Didn't Believe Me, mes. 1-10.

They Didnit Believe Me.
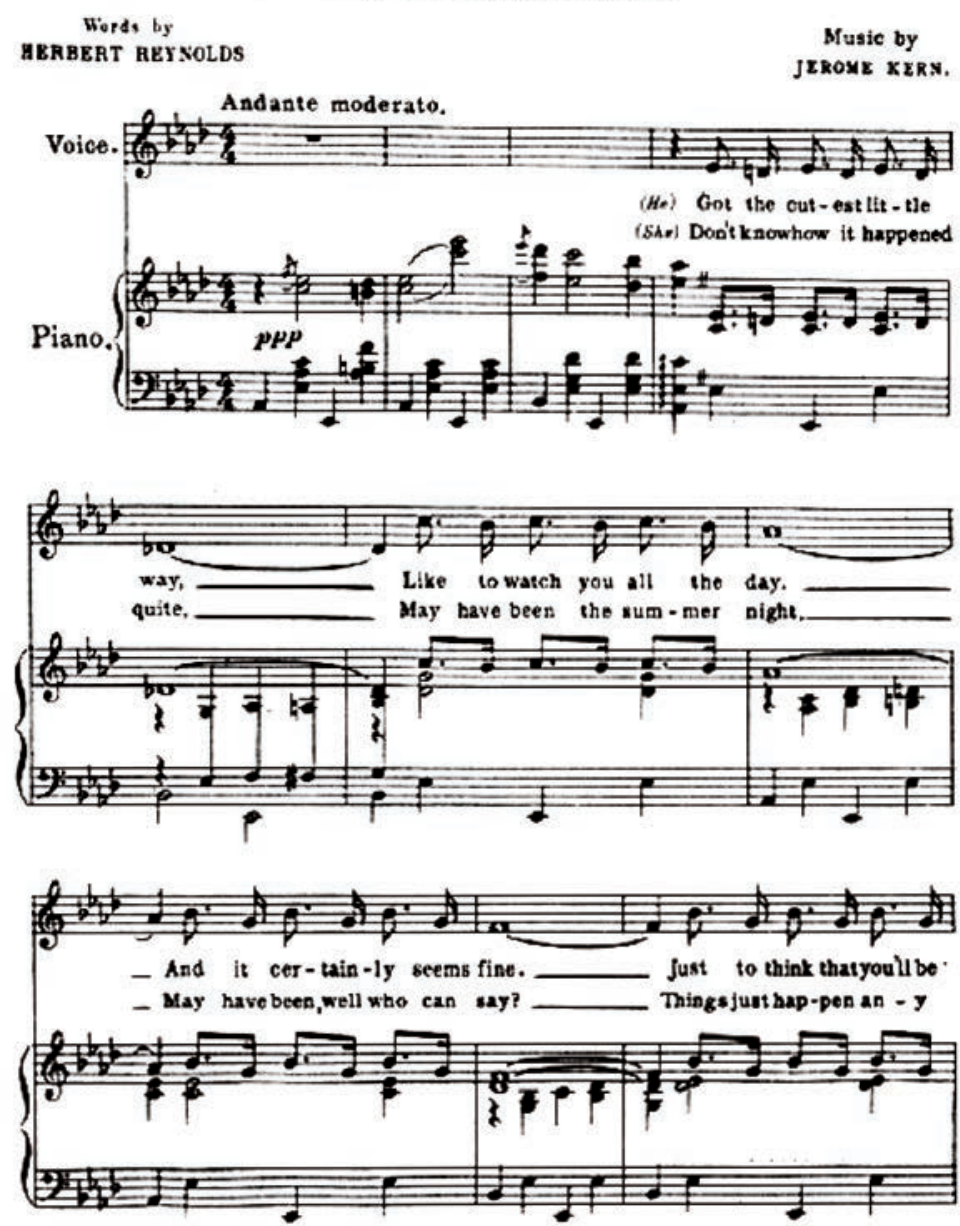

CopyrigAt wCNXIV by T. B. Harme \& Preacio, Day \& Nunter, N.Y

soso- 4 All stage rights reserved by the Composet. taterationat Cepyrigat Secuted.

structure simple, aisément perceptible, qui fait se succéder de manière régulière, voire systématique, des carrures de quatre mesures, et par le rythme pointé de la mélodie, qui distinguait alors le fox-trot des autres genres musicaux associés au jazz.

Ces quatre caractéristiques se retrouvent dans Adieu NewYork! d'Auric. Ce dernier semble donc avoir fait en sorte que sa musique puisse être identifiée clairement à un foxtrot. Mais en même temps, tout se passe comme si le genre auquel la pièce fait référence était malicieusement perverti

\footnotetext{
Il ressort de la correspondance entre Auric et Cocteau (son mentor à la fin des années 1910) que le projet de cette pièce, d'abord conçu comme un ballet de plus grande envergure, remontait au mois de janvier 1918 (Caizergues 1999).

6 Pour ne prendre qu'un exemple de l'irruption du jazz sur la scène parisienne, à la fin de la Première Guerre mondiale, sa présence dans la revue Laissez-les tomber, créée le 12 décembre 1917 au Casino de Paris, fait grand bruit dans la capitale à tel point que Cocteau en livre une description savoureuse dans Le Coq et l'Arlequin (Cocteau 1918, 29).

7 Les disques et les musiciens américains ont également joué un rôle important dans la diffusion du jazz en France. Il n'en reste pas moins vrai qu'en 1919 et en 1920, les partitions de fox-trot, de blues et de ragtime distribuées, entre autres par les éditions Salabert et Max Eschig, sont les principales sources citées par les compositeurs français.

8 Composé par Irving Berlin sur des paroles de Ted Synder en 1911, That Mysterious Rag fut publié la même année et exporté au Canada et en Europe.

9 La Tin Pan Alley était située à New York, au niveau de la $28^{\mathrm{e}}$ rue, entre les $5^{\mathrm{e}}$ et $6^{\mathrm{e}}$ avenues. Elle rassemble jusque dans les années 1930 des maisons d'édition musicale qui fournissaient aux États-Unis un vaste répertoire de chansons populaires.
} 
Figure 2: Georges Auric, Adieu New-York!, mes. 1 à 21.

is Jean COCTEAU

\section{Adied, New-York! FOX-TROT}

Georger AUIIC
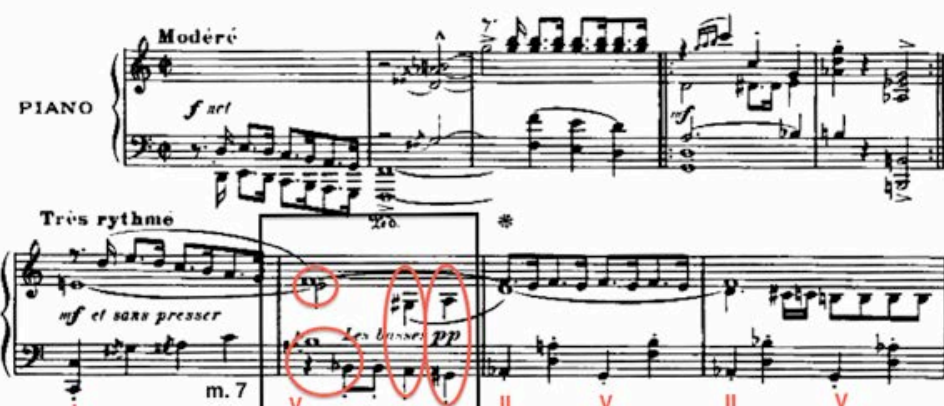

m.7 $v$ (

II
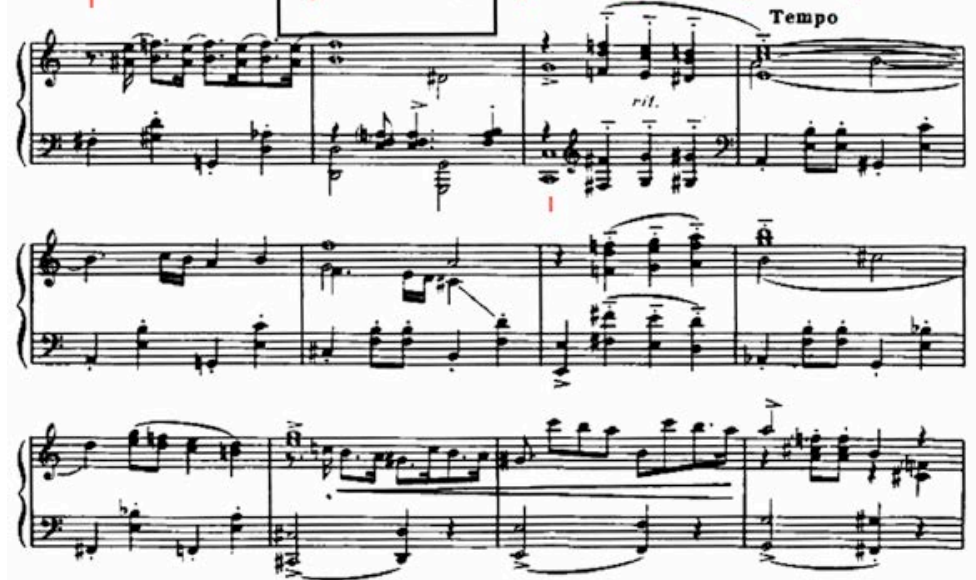

par l'adjonction de dissonances ${ }^{10}$. À travers cette esthétique de la fausse note ${ }^{11}$, tout porte à croire en effet qu'Auric s'est amusé à introduire au sein d'un langage tonal fonctionnel des erreurs volontaires.

Ainsi, dans la seule mesure 7 d'Adieu New-York! (figure 2), Auric accumule une dissonance créée par l'attaque d'un $f a$ à la main droite, qui se heurte à la résonnance du $m i$ de la mesure précédente (mes. $7^{1}$ ), une fausse relation entre le $s i$ bécarre et le $s i$ bémol à la main gauche (mes. $7^{1-2}$ ), puis un échange volontairement dissonant entre le sol dièse et le la à la main gauche (mes. $\left.7^{2-3}\right)$.

Une telle déformation volontaire du langage tonal du foxtrot fait de la pièce d'Auric une page légère et farceuse. Cette déformation permet de mieux comprendre la portée esthétique d'une telle pièce en 1919. Encore faut-il pour cela la resituer dans le champ de la création musicale savante française de l'époque.

\section{Adieu New-York!: une illustration musicale du Coq et l'Arlequin de Jean Cocteau}

En composant Adieu New-York!, Auric rompt avec les sources d'inspirations privilégiées et avec certaines conventions musicales en vigueur dans la France de la fin des années 1910. L'œuvre renvoie notamment dos à dos deux courants esthétiques alors majeurs dans la musique française: le wagnérisme et le debussysme ${ }^{12}$. À l'univers légendaire et sublime des œuvres wagnériennes et aux aspirations hautement poétiques et symbolistes du debussysme, Adieu New-York! oppose ainsi une musique dont la source d'inspiration, bien moins élevée, relève de l'univers du quotidien et du divertissement: celui du jazz de music-hall.

Plus généralement, Adieu New-York! prend à revers la conception romantique de la musique populaire: celle d'une musique issue d'un univers rural non encore perverti par la civilisation et considérée comme anhistorique car incarnant l'essence d'un peuple ${ }^{13}$. Le caractère populaire du foxtrot est en effet tout autre: il fait référence à une musique destinée au plus grand nombre, issue d'un univers urbain, celui des théâtres et des ballrooms, et enfin à une musique non plus anhistorique mais clairement datée ${ }^{14}$ puisque les premiers fox-trots furent composés et publiés au début des années 1910.

Adieu New-York! peut donc être considéré comme une pièce rompant avec les référents esthétiques du debussysme, du wagnérisme et plus généralement d'un romantisme

10 Dès 1923, Milhaud fait remarquer qu'Auric ne cherchait pas à composer un fox-trot en bonne et due forme. Il affirme que «dans Parade de M. Érik Satie, ou Adieu New-York de M. Georges Auric (...), nous avons le portrait d'un rag-time ou d'un fox-trot» (Milhaud 1923, 163; c'est moi qui souligne).

11 J'emploie cette expression car le procédé d'Auric est utilisé de manière systématique et volontaire dans cette pièce. D'autre part, on le retrouve dans d'autres œuvres et chez d'autres compositeurs: la troisième et la cinquième des Cinq pièces faciles de Stravinski (1917), ou encore Pulcinella du même compositeur (1919).

12 Témoin de l'évolution du monde musical français après la Grande Guerre, l'historiographe René Dumesnil cite par exemple ces deux courants («debussysme» étant remplacé par «impressionnisme») dès le premier chapitre, «Courants et tendances avant et après 1914 », de La Musique en France entre les deux guerres (Dumesnil 1946, 13). Le 20 mai 1902, le critique musical du Temps, Pierre Lalo, évoque un "wagnérisme obligatoire» (Lalo 1902, 3). Quand à l'esthétique de Claude Debussy, celle plus précisément de Pelléas et Mélisande, elle marqua si fortement la musique française que le musicographe Paul Landormy plaça son histoire de la musique dans l'entre-deux-guerres sous son patronage en l'intitulant $L a$ musique française après Debussy (1943). Toutefois, Debussy s'amusa lui-même à désacraliser le Sublime wagnérien dans "Golliwog's Cakewalk» (1908), où il traite le leitmotiv de Tristan und Isolde à la manière d'un ragtime.

13 Cette conception romantique de la musique populaire a notamment été analysée par l'historienne Anne-Marie Thiesse dans La création des identités nationales (1999).

14 En cela, le fox-trot correspond aux caractéristiques de la musique de masse telle que les identifie l'historien Ludovic Tournès dans La culture de masse en France de la Belle Époque à aujourd'hui (Tournès dans Sirinelli 2002, 220-258). 
musical au sens large qu'incarnent encore, en 1920, un Richard Strauss ou, en France, un Vincent d'Indy ${ }^{15}$.

La même prise de distance peut également être observée dans les caractéristiques musicales d'Adieu New-York!. À la subtilité formelle des œuvres majeures de Debussy, à la souplesse de ses phrases et à celle de leur harmonie, Auric répond par une pièce nettement segmentée et caractérisée par une carrure parfaitement régulière dans un contexte tonal fonctionnel. De même, à la tonalité poussée dans ses derniers retranchements par le Tristan und Isolde (1865) de Wagner, à la modalité sensuelle du Pelléas et Mélisande (1902) et de la «Cathédrale engloutie» (1910) de Debussy, Auric oppose une tonalité fonctionnelle relativement simple. Quant à la profondeur de la musique wagnérienne et à l'univers mystérieux de la musique debussyste, Auric leur oppose une musique franchement joviale, espiègle et farceuse au point de mettre en scène ses propres fausses notes. Au vu des caractéristiques d'Adieu New-York! et de la date de la composition de cette pièce étonnante, celle-ci peut donc être considérée comme une traduction musicale des positions esthétiques affirmées dans Le Coq et l'Arlequin de Cocteau (1918).

Dans ce bref pamphlet dédicacé, de manière significative, à Auric, le futur promoteur du Groupe des Six définit de manière polémique la nécessité de renvoyer dos à dos debussysme et wagnérisme pour refonder une esthétique à la fois moderne et française:

Le music-hall, le cirque, les orchestres américains de nègres, tout cela féconde un artiste au même titre que la vie [...]. On se demande pourquoi Satie affuble ses plus belles œuvres de titres drôles [...]. Outre que ces titres protègent son œuvre des personnes en proie au «sublime», ils s'expliquent par l'abus debussyste des titres précieux [...]. Satie nous enseigne la plus grande audace à notre époque: être simple [...]. Il déblaie, il dégage, il dépouille le rythme (Cocteau 1918, 56, $60,63)$.

La communauté d'idées esthétiques qui unissait alors l'écrivain et le jeune compositeur apparaît parfaitement dans un article d'Auric paru dans l'éphémère revue Le Coq, dirigée par Cocteau lui-même ${ }^{16}$ :

Aimant voir clair, nous répugnons au mensonge $\mathrm{du}$ «sublime», à l'engourdissement fakirique des «temples qui furent». Jean Cocteau a raison: «toute affirmation profonde nécessite une négation profonde » [...]. Ayant grandi au milieu de la débâcle wagnérienne et commencé d'écrire parmi les ruines du debussysme, imiter Debussy me paraît aujourd'hui la pire forme de la nécrophagie [...]. Depuis nous avons eu le cirque, le music-hall et les orchestres américains. Tout cela nous a réveillés. Mais, Adieu New-York !... $(1920,1)$.

Trois remarques peuvent être formulées à propos de ces deux extraits. Les attaques contre le «sublime» et les «titres précieux» révèlent tout d'abord une volonté exprimée de manière explicite de sortir la musique française de l'influence de Wagner et de Debussy. Deuxièmement, de nouvelles sources d'inspirations sont proposéesafin de se dégager de cette influence: le «cirque», le «musichall», les «orchestres américains de nègres», soit celles exactement dont Auric tire son fox-trot. Enfin, Auric et Cocteau, à travers les références à Satie, mettent à distance le romantisme en situant leur esthétique iconoclaste dans une mouvance néo-classique alors en vogue ${ }^{17}$, dont on retrouve les caractéristiques dans la simplicité de la forme et du langage ainsi que dans le caractère léger et farceur d'Adieu New-York!. Ce n'est donc pas par hasard si Satie, le compositeur cité comme une référence contemporaine pour Cocteau, figure également, avec Stravinski, au panthéon d'Auric à la fin des années 1910 (Auric 1979, 47, 50).

\section{La place d'Adieu New-York! dans le monde musical français}

Le fox-trot du jeune compositeur français montre donc que le jazz avec lequel il a pu être en contact dans la France de la fin des années 1910 a bel et bien été détourné en ce qu'il n'est pas convoqué sans arrière-pensées. Volontairement déformé, il permet à Auric de produire une œuvre dont les caractéristiques musicales et les connotations extramusicales se distinguent radicalement, et de manière provocatrice, du wagnérisme et du debussysme. Ce faisant, comme le résume le schéma suivant (figure $3^{18}$ ), Auric rejoint les positions et le goût de l'avant-garde musicale française de l'époque.

Indépendamment de la référence au jazz, le caractère et les traits stylistiques d'Adieu New-York! placent effectivement Auric dans le sillage de Satie (plus particulièrement d'une de ses œuvres majeures, Parade) et dans celui du Stravinski néo-classique de la fin des années 1910, qui utilise la même esthétique de la fausse note dans plusieurs de ses œuvres de cette période ${ }^{19}$.

C'est donc la signification même du jazz qui fait l'objet d'un détournementdans Adieu New-York!: musique de

\footnotetext{
15 La manière dont ce dernier utilise le folklore musical français dans des ouvres comme les Chants du terroir (1918) correspond parfaitement avec les éléments relevés par Anne-Marie Thiesse. Au début des années 1920, d'autres compositeurs s'inscrivent dans cette veine folkloriste, comme Joseph Canteloube et ses Chants d'Auvergne (1924).

16 Comme l'indique le titre de cette revue, son orientation éditoriale se situe dans la droite ligne du plaidoyer pour un renouveau de la musique française formulé deux ans plus tôt par Cocteau dans Le Coq et l'Arlequin.

17 Voir à ce sujet Messing 1988 et 1991.

18 Les ovales barrés comprennent le nom des institutions, des courants ou des compositeurs auxquels s'opposa explicitement Auric.

19 Voir la note 11
} 
Figure 3: Le contexte artistique de la création d'Adieu New York!

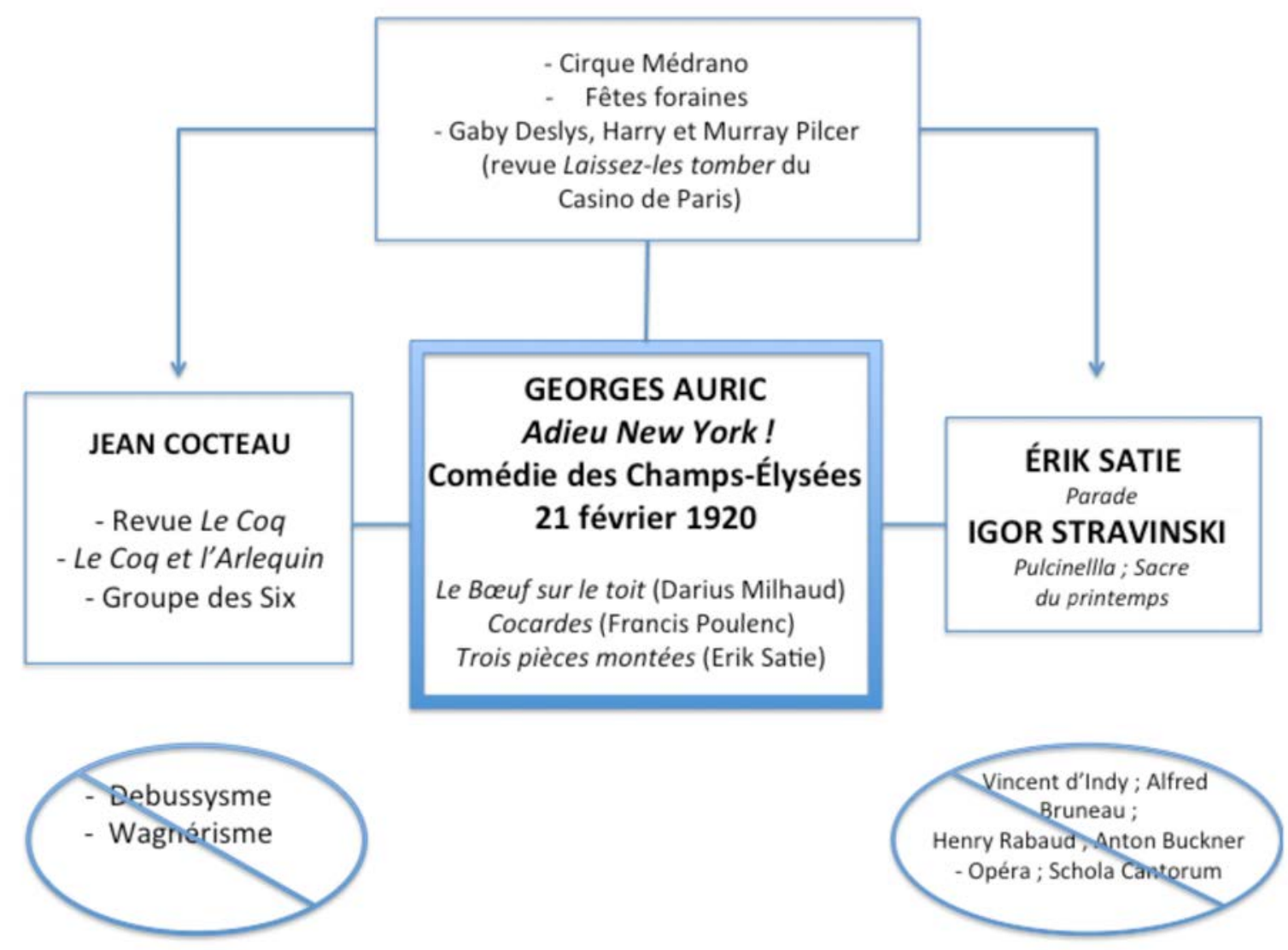

divertissement à la mode et jouée sur les scènes de musichall parisien, le jazz sert à Auric de marchepied pour apparaître comme un jeune compositeur savant à la pointe de la modernité dans les débats autour du debussysme et du wagnérisme qui agitent alors la France depuis les années $1900^{20}$. Une fois importés dans le domaine de la musique savante, le jazz et les représentations sulfureuses qu'il véhicule alors permettent en outre à Auric de faire scandale et, par là, de se faire connaître du public.

Un tel détournement du jazz ne peut donc se comprendre qu'en tenant compte du jazz qu'Auric a pu entendre, mais aussi, et surtout, des différents courants esthétiques à l'œuvre dans le monde musical français, auxquels sa pièce s'adosse ou s'oppose. La manière de traiter le jazz dans Adieu NewYork! semble donc dépendre en partie des problématiques à l'œuvre dans l'univers musical au sein duquel Auric composa cette pièce. Afin de valider cette hypothèse, il reste à se pencher sur une œuvre savante recourant elle aussi au jazz, mais produite dans un tout autre contexte musical: le «Ragtime» de la Suite 1922 de Paul Hindemith.

\section{Paul Hindemith et la parodie expressionniste du ragtime}

De même qu'en France, le jazz fut diffusé en Allemagne, au début des années 1920, par quatre vecteurs principaux: la circulation des musiciens dès 1919 (Wipplinger 2007 et Lotz 2006), l'édition de partitions, l'édition de disques (Lotz 2006) et la radiodiffusion (Lotz 2008). Dès les années 1910, Paul Hindemith a pu se familiariser avec un répertoire de musique alors largement constitué de ragtimes, lorsqu'il jouait dans des cafés de Francfort pour soutenir financièrement sa famille après le décès de son père ${ }^{21}$. Parmi les plus célèbres de ces partitions figurait Original Rags de Scott Joplin 22 .

Outre la pompe de la main gauche ${ }^{23}$, le langage tonal fonctionnel et la régularité parfaite des phrases, constituées de groupes de quatre ou de huit mesures, deux caractéristiques saillantes du ragtime peuvent être identifiées dès les premières mesures de cette pièce. D'une part, le rythme des syncopettes et des groupes de quatre doubles croches liées

\footnotetext{
${ }^{20}$ Le jazz fut en effet, dès les années 1920, le signe de ralliement des avant-gardes musicales, comme le montre la figure 3, mais également littéraire puisqu'il attira l'attention du groupe Surréaliste.

21 Celui-ci fut tué pendant la Première Guerre mondiale.

22 Pionnier de ce genre musical dès le début des années 1890, Scott Joplin (1867-1917) vit sa musique rapidement diffusée en Europe (y compris en Allemagne).

23 Voir l'exemple encadré de la figure 4 (mes. 12-13).
} 
Figure 4: Scott, Joplin, Original Rags, mes. 1 à 25.

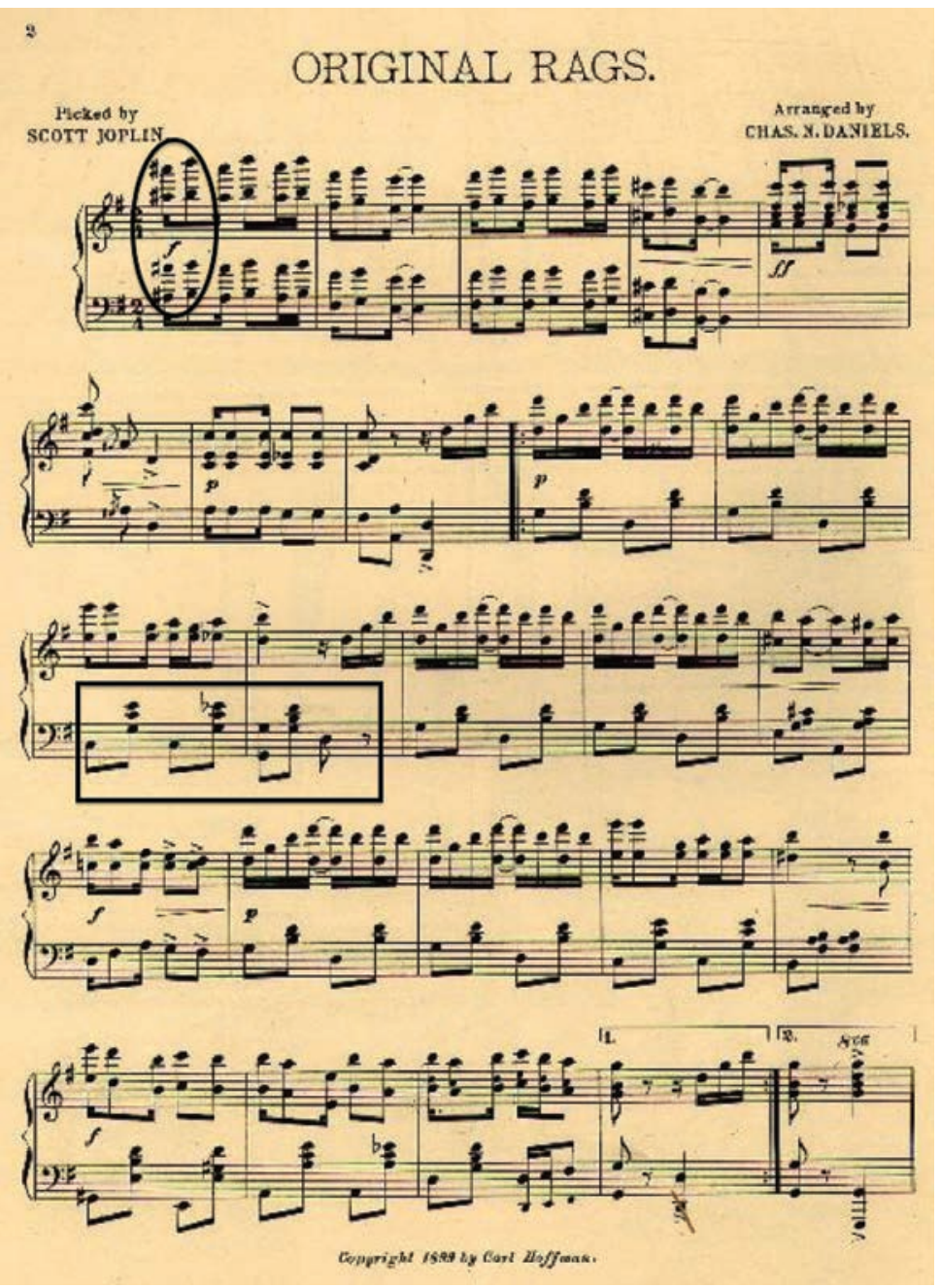

l'un à l'autre ${ }^{24}$ est d'autant plus efficace qu'il contraste avec la régularité presque mécanique de la pompe. D'autre part, comme dans la plupart des ragtimes, Original Rags s'ouvre sur une introduction en homorythmie qui s'achève sur une harmonie de dominante ${ }^{25}$.

À ces éléments s'en ajoute un autre, caractéristique, quant à lui, du blues: Joplin joue dès le début d'Original Rags sur l'ambiguiité, dans la tonalité de sol, de la tierce, tantôt mineure ( $s i$ bémol, ou la dièse par enharmonie), tantôt majeure (si bécarre). Il s'agit ici de la blue note que les compositeurs savants de l'époque ne manquèrent pas de remarquer ${ }^{26}$.

\footnotetext{
24 Voir dans la figure 4, la portée supérieure des mesures 1-4 et 9-10.

25 Aux mesures 1-8 (figure 4).

26 Voir l'exemple entouré de la figure 4 (mes. 1).

27 Voir les mesures 4-5 et 9-10 de la figure 5.

28 Voir dans la figure 5, la portée inférieure des systèmes à partir de la mesure 9.

29 Voir les mesures $1-3^{1}$ de la figure 5.

30 Soit do-ré-mi-fa-sol-la-si contre do dièse-ré dièse-fa dièse-sol dièse-la dièse. Voir la mesure 8 de la figure 5.

31 Voir par exemple, aux mesures 1 et 2, les superpositions de quartes frappées sur les temps et les contretemps.

32 Voir, aux mêmes mesures, les septièmes frappées en alternance avec les empilements de quartes. Hindemith va même jusqu'à combiner les deux procédés qui viennent d'être mentionnés: à la mesure $6^{2}$, par exemple, deux quartes, mi bémol-la bémol et sol bémol-do bémol, sont attaquées simultanément à distance d'une septième mineure.
} ragtime, ni à en composer un pastiche: une fois encore la musique de jazz est déformée. Mais cette déformation n’est pas, dans la Suite 1922, de même nature que celle que fait subir Auric au fox-trot; tout se passe ici comme si Hindemith brossait le portrait d'un ragtime rendu sauvage, difforme et, en somme, quasiment inhumain.

Mais pour que ce portrait puisse être reconnaissable par le public allemand de l'époque, encore faut-il maintenir assez de références musicales au ragtime usuel. C'est ainsi que Hindemith maintient la plupart de ses caractéristiques: le rythme syncopé de la main droite ${ }^{27}$ (qui est le même, par ailleurs, que celui employé dans Original Rags); la pompe de la main gauche ${ }^{28}$ et l'introduction en homorythmie qui s'achève sur un accord remplissant une fonction de dominante ${ }^{29}$. Ce sont donc principalement les caractéristiques rythmiques du ragtime (celles que les commentateurs allemands de l'époque identifiaient comme les plus caractéristiques de ce genre (Cohen-Avenel 2011, 123-175)) que reprend à son compte Hindemith. En revanche, le langage tonal fonctionnel du ragtime se voit profondément déformé.

\section{La dissonance comme dissolvant de la tonalité}

Hindemith substitue en effet au langage tonal fonctionnel une tonalité ouverte, organisée autour d'un pôle: le do dièse. Celui-ci est affirmé de manière forte à la fin de l'introduction, où il sonne comme une résolution (mes. $4^{1}$ ), au retour du premier thème (mes. $9^{1}$ ) ou encore à la fin de la pièce. À partir de cette polarité, Hindemith déploie un langage extrêmement dissonant grâce à trois procédés: la superposition des échelles formées par les touches noires et par les touches blanches (échelle de do contre le mode pentatonique sur $d o$ dièse $^{30}$ ); l'usage d'empilements de quartes qui donnent une sonorité assez farouche à cette page $^{31}$; et par des secondes ou des septièmes frappées

Quant au jeu sur les tierces majeures et mineures signalé dans Original Rags, Hindemith le reprend à son compte mais en détourne la fonction: au lieu de sonner comme une coloration évoquant une blue note, l'ambiguïté entre $m i$ dièse et $m i$ bécarre (tierces majeure et mineure de $d o$ 
Figure 5: Paul Hindemith, Suite 1922, «Ragtime», mes. 1 à 18.

\section{$\mathrm{V}$}

Ragtime
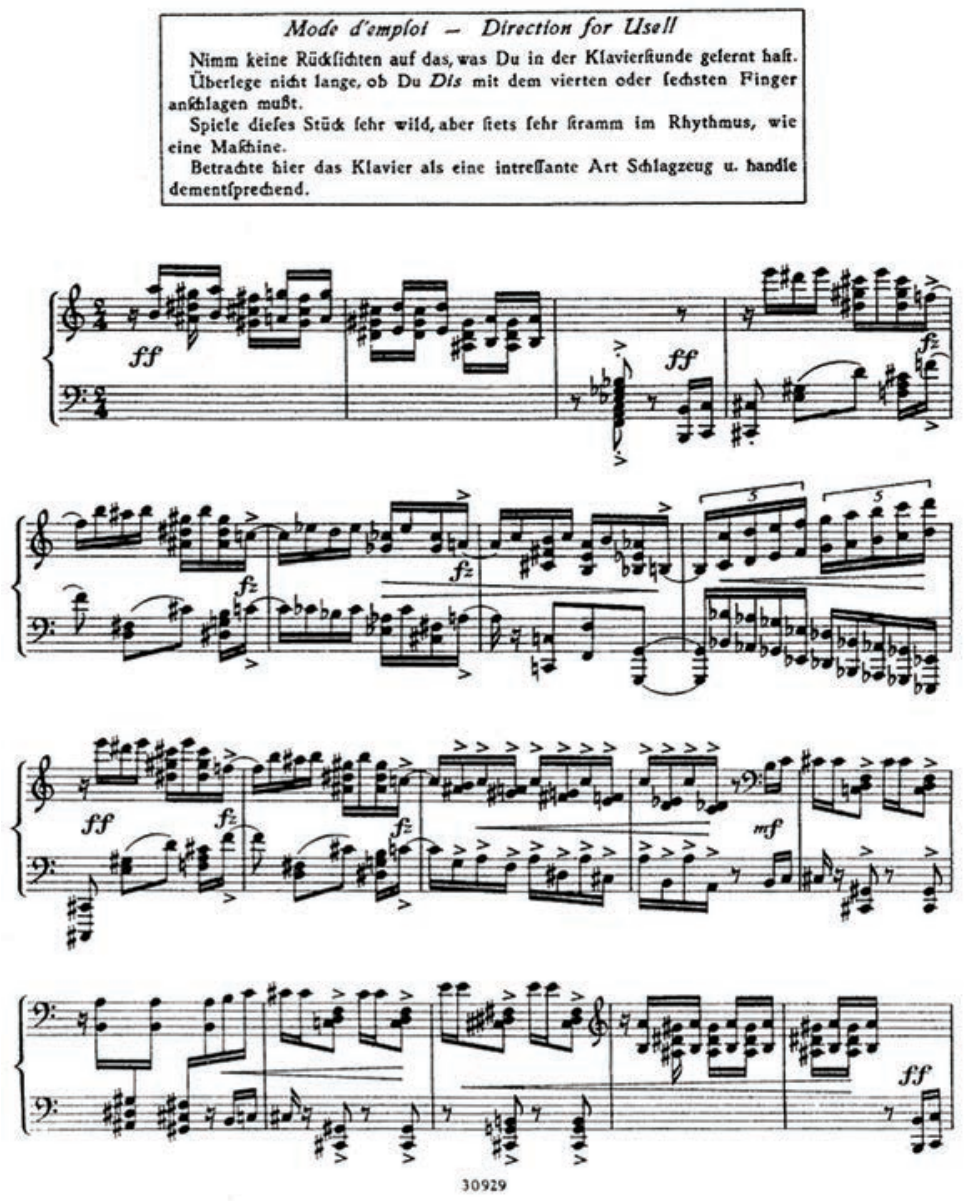

dièse) contribue au caractère violemment dissonant de la pièce. Ainsi, quand la logique mélodique de la première phrase (qui commence à la mesure $4^{1}$, à la portée supérieure) présage une résolution sur un sol dièse ou un $m i$ bécarre (mes. $4^{2}$, dernière double croche ${ }^{33}$ ), Hindemith écrit plutôt un $f a$ bécarre (ou un $m i$ dièse, par enharmonie) d'autant plus surprenant qu'il est frappé à l'octave par les deux mains, accentué et marqué par un sforzando (mes. $4^{2}$, dernière double croche).

\section{De la violence sonore}

La déformation des traits du ragtime ne se limite cependant pas à une transformation de l'aimable tonalité fonctionnelle du ragtime traditionnel en un langage sombre, aux violentes dissonances. Au moyen de dynamiques exacerbées qui, d'un bout à l'autre du morceau, ne descendent jamais sous le fortissimo, et grâce à une doublure systématique à l'octave des basses de la main gauche, Hindemith fait de son ragtime un véritable coup de poing sonore. Le «mode d'emploi ${ }^{34}$ » qu'il fait figurer en tête du morceau confirme cette intention de faire du ragtime une page musicale violente:

Oublie tout ce que tu as appris lors de tes cours de piano. Ne te soucie pas de savoir si tu dois jouer le ré dièse avec le quatrième ou le sixième doigt. Joue cette pièce avec férocité, mais strictement en rythme, comme une machine. Considère le piano comme un instrument à percussion intéressant, et traite-le en conséquence ${ }^{35}$ (Hindemith 1922, 19).

L'utilisation de l'expression «mode d'emploi» est assez insolite pour que l'on s'y arrête. Ce petit texte, qui témoigne sans doute du regard espiègle que jette Hindemith sur sa pièce, remplace les indications d'ordre expressif traditionnellement placées en tête d'un morceau ${ }^{36}$. Le «Ragtime» apparaît donc moins comme une pièce musicale à interpréter que comme un objet sonore à produire. Tout se passe comme si Hindemith mettait en scène une musique qui aurait troqué l'homme pour la machine.

À travers cette page exacerbée, Hindemith annonce, au second degré certes, une position récurrente dans les débats suscités par l'arrivée et la vogue du jazz en Allemagne dès le début des années 1920. Cette position est notamment développée par Siegfried Kracauer dans deux essais, «Die Revuen» (1925) et «Das Ornament der Masse» (1927). Le jazz y est considéré comme un symptôme de la mécanisation de la société allemande dans laquelle l'homme disparaitrait au profit de la machine, la sensualité au profit du sexe, et le sentiment humain au profit de l'argent ${ }^{37}$.

\section{Pour une "esthétique du laid»: Un ragtime expres- sionniste}

Le «Ragtime» de Hindemith part donc des caractéristiques de son modèle pour le détourner et en faire une page de musique monstrueuse et déshumanisée (au sens où elle met en scène la disparition de tout sentiment humain). Si l'on retrouve dans cette pièce l'esprit farceur et iconoclaste d'Adieu New-York!, les deux pièces diffèrent en revanche sur le plan stylistique: à l' «esthétique de la fausse note» d'Auric, Hindemith répond par une «esthétique du laid». Ce choix prend tout son sens si l'on tient compte de sa

\footnotetext{
33 Une oreille formée au langage tonal fonctionnel attendrait en effet une résolution sur la tierce ou la quinte de $d o$ dièse.

34 Voir la figure 5.

35 «Nimm keine Rücklichten auf das, was Du in der Klavierstunde gelernt hast. Überlege nicht lange, ob Du Dis mit dem vierten oder sechsten Finger anschlagen musst. Spiele dieses Stück sehr wild, aber stets sehr stramm im Rhythmus, wie eine Maschine. Betrachte hier das Klavier als eine intressante Art Schlagzeug u. handle dementsprechend». Traduction personnelle.

36 Une fois encore, Hindemith travaille à déjouer les attentes des mélomanes.

37 Plus généralement, Kracauer décrivait en effet le jazz comme un « exutoire ou un défoulement provisoire après la guerre» (Cogen-Avenel 2011, 153). «Lorsque Kracauer décrit les jambes des girls comme des machines, il ne s'agit pas d'une comparaison, ni même d'une métaphore: elles sont bel et bien un produit industriel de série fabriqué à la chaîne» (Cohen-Avenel 2011, 163).
} 
Figure 6: Otto Dix, Metropolis (1928).
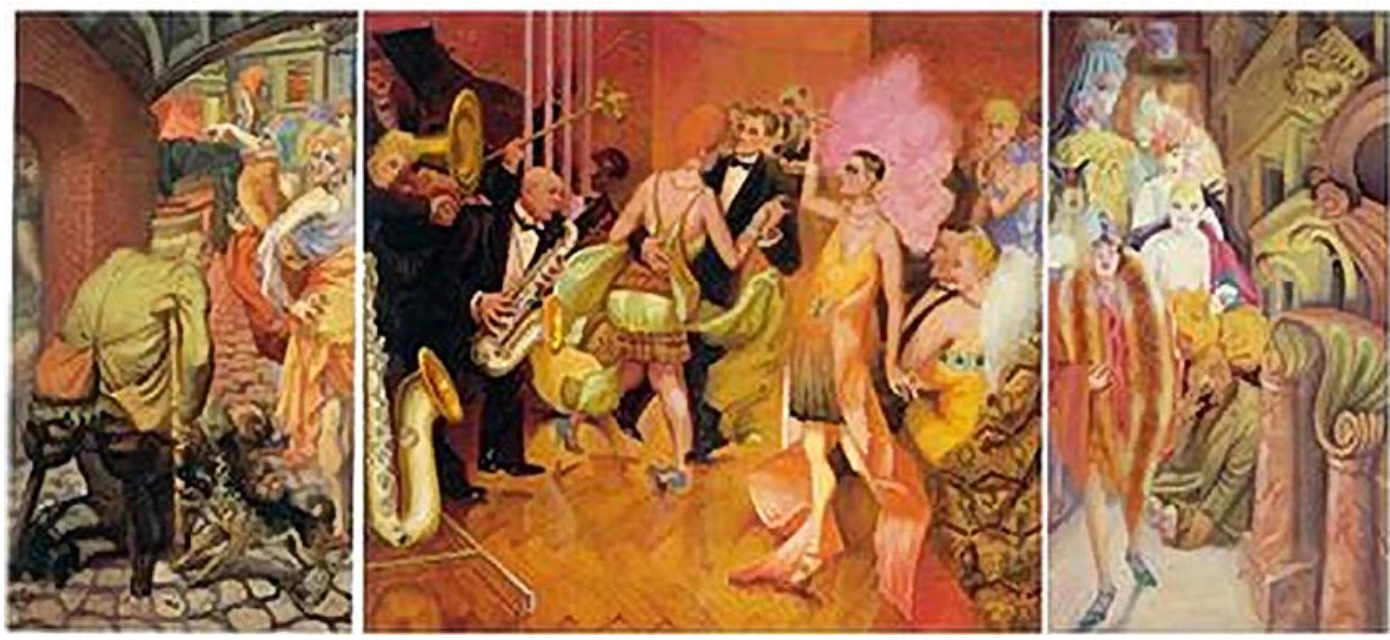

proximité, en 1919, avec les cercles expressionnistes allemands. Au moment où il compose la Suite 1922, Hindemith a mis en musique (ou s'apprête à le faire) des livrets d'opéras écrits par des écrivains phares de ce milieu comme Oscar Kokoschka, August Stramm, Franz Blei et Georg Trackl ${ }^{38}$.

Le «Ragtime» de Hindemith peut ainsi être rapproché, tant sur le plan du contenu expressif que sur celui de la démarche artistique $^{39}$, de Metropolis (figure 6), chefd'œuvre de l'un des peintres phares de l'expressionnisme pictural: Otto Dix.

Dans ce tableau, comme dans «Ragtime», la musique de jazz fait partie d'une vision sombre: celle de la déliquescence de la société allemande que la Première Guerre mondiale, symbolisée dans le volet gauche du triptyque par un soldat mutilé, aurait rendue sauvage et vulgaire. Ainsi juxtaposé avec la Grande Guerre, le jazz se voit en effet associé avec la déshumanisation. L'amour, sentiment humain par excellence, a cédé la place, dans les trois volets du triptyque, au commerce du sexe symbolisé par la présence de prostituées et de demi-mondaines dans les bas-fonds où se produisent les musiciens de jazz, et dont la vulgarité est soulignée par la teinte rouge criarde qui domine le panneau central $^{40}$.

Au terme de ces deux études de cas, il semble bel et bien possible de dégager des spécificités nationales de la réception du jazz. La singularité d'Adieu New-York! et du «Ragtime» de la Suite 1922 résulte en effet des courants esthétiques à l'œuvre, respectivement en France et en Allemagne à la fin des années 1910 et au début des années 1920.
Alors qu'Auric détourne certaines caractéristiques du fox-trot afin de s'inscrire dans le sillage de Cocteau et de prendre position dans les débats concernant la redéfinition de l'identité française de la musique ${ }^{41}$, les caractéristiques du ragtime sont détournées par Hindemith pour donner une image sonore ironique de la crise de l'identité et de la civilisation allemandes au lendemain de la Première Guerre mondiale.

En dernière analyse, l'étude de ces deux compositions, qui met en résonnance des éléments purement musicaux avec des éléments tirés de leur contexte esthétique culturel, permet de comprendre comment la réception d'une expression culturelle étrangère (en l'occurrence, le jazz), bien loin d'être passive, est productrice de significations originales qui proviennent en partie du contexte d'accueil de ces expressions. En d'autres termes, le type d'analyse qui vient d'être proposé contribue à démontrer que notre manière de percevoir et de nous approprier ce qui se présente comme une altérité est tributaire de ce qui constitue notre propre identité et des débats autour de sa définition. Et une part de cette identité semble bel et bien relever de l'appartenance nationale.

38 Librettistes respectifs de Mörder, Hoffnung der Frauen (1919), Sancta Susanna (1921), Das Nusch-Nuschi (1920) et Die Junge Magd (1922).

39 Celle consistant à exacerber certains traits caractéristiques du modèle auquel une pièce ou une toile fait référence.

40 Il n'est pas question ici d'analyser plus à fond cette toile. Il convient au demeurant d'ajouter que le recours au triptyque, objet initialement destiné à orner les autels des Églises, n'a rien d'anodin: le sacré se voit ici remplacé par le divertissement profane, la foi par l'argent.

41 Une identité débarrassée de l'influence allemande incarnée par Richard Wagner, et le l'influence russe qu'aurait reprise à son compte l'impressionnisme musical de Debussy (Cocteau 1918). 


\section{RÉFÉRENCES}

Auric, Georges (1919). Adieu New-York!, Paris, Éditions de la Sirène. Partition de piano.

Auric, Georges (1920). «Bonjour Paris!», Le Coq, nº 1 , avril, p. 1 .

Budds, Michael J. (2002). Jazz and the Germans: Essays on the Influence of "hot» American Idioms on the 20thCentury German Music, Hillsdale, Pendragon Press.

Caizergues, Pierre (1999). Jean Cocteau, Georges Auric: Correspondance, Montpellier, Presses Universitaires de la Méditerranée.

Cocteau, Jean (1918). Le Coq et l'Arlequin: Notes autour de la musique, Paris, Éditions de la Sirène.

Cohen-Avenel, Pascale (2011). Si on a du jazz, pas besoin de schnaps: Jazz, négritude et démocratie sous la République de Weimar, Francfort, Peter Lang.

Dumesnil, René (1946). La musique en France entre les deux guerres, 1919-1939, Paris, Éditions du Milieu du Monde.

Gendron, Bernard (2002). Between Montmartre and the Mudd Club, Chicago, University of Chicago Press.

Guerpin, Martin (2012). «Why Did Art Music Composers Pay Attention to Jazz? The Impact of Jazz on the French musical Field (1908-1927) », dans Luca Cerchiari, Laurent Cugny et Franz Kerbschbaumer (dir.), Eurojazzland, Jazz and European Sources, Dynamics and Context, Boston, Northeastern University Press, p. 47-80.

Hindemith, Paul (1922). Suite 1922, Mayence, Schott. Partition de piano.

Hodeir, André (1954). Hommes et problèmes du jazz, Marseille, Parenthèses.

Joplin, Scott (1899). Original Rags, Kansas City, Carl Hoffman. Partition de piano.

Kern, Jerome (1914). They didn't Believe Me, New York, Harms and Francis Day. Partition chant et piano.

Kracauer, Siegfried (1925/1971). «Die Revuen», dans Siegfried Kracauer, Schriften, Francfort-sur-le-Main, Suhrkamp, vol. 5, tome 1, p. 338-342.

Kracauer, Siegfried (1927/1971). «Das Ornament der Masse», dans Siegfried Kracauer, Schriften, Francfortsur-le-Main, Suhrkamp, vol. 5, tome 2, p. 57-68.

Lalo, Pierre (1902). «La Musique», Le Temps, 20 mai, p. 3.

LANDORMy, Paul (1943). La musique française après Debussy, Paris, Gallimard.

Lotz, Rainer (2006). Deutsche Hot-Discographie: CakeWalk, Ragtime, Hot Dance und Jazz - Ein Handbuch, Bonn, Birgit Lotz Verlag.
Lotz, Rainer (2008). Der Jazz im Deutschland: Von Cake Walk zum Jazz, Boston-Quartett et al., Bear Family Records, BCD 16909, 3 disques compacts.

Mawer, Deborah (2010). «Crossing Borders II: Ravel's Theory and Practice of Jazz», dans Deborah Mawer (dir.), Ravel Studies, Cambridge, Cambridge University Press, p. 114-137.

Messing, Scott (1988). Neoclassicism in Music: From the Genesis of the Concept through the Schoenberg/ Stravinsky Polemic, Rochester, University of Rochester Press.

Messing, Scott (1991). «Polemic as History: The Case of Neoclassicism», The Journal of Musicology, vol. 9, $\mathrm{n}^{\circ} 4$, p. 481-497.

Milhaud, Darius (1923). «L'évolution du jazz-band et la musique des nègres d'Amérique du Nord», Courrier Musical, vol. 25, n 9, p. 163-164. Repris dans Darius Milhaud (1982), Notes sur la musique: Essais et chroniques, Paris, Flammarion, coll. «Harmoniques», p. 102.

Perloff, Nancy (1991). Art and the Everyday: Popular Entertainment and the Circle of Erik Satie, New York, Oxford University Press.

Perret, Carine (2003). «L'adoption du jazz par Darius Milhaud et Maurice Ravel: L'esprit plus que la lettre», Revue de musicologie, vol. 89, p. 311-347.

Ross, Alex (2007). The Rest is Noise: Listening to the Twentieth Century, New York, Picador.

SChUller, Gunther (1968). Early Jazz: Its Roots and Musical Development, New York, Oxford University Press.

Thiesse, Anne-Marie (1999). La création des identités nationales, Paris, Éditions du Seuil.

TouRnÈs, Ludovic (2002). «Reproduire l'œuvre: La nouvelle économie musicale», dans Jean-Pierre Roux et Jean-François Sirinelli (dir.), La culture de masse en France, de la Belle Époque à aujourd'hui, Paris, Fayard, p. 220-258.

WIENER, Jean (1978). Allegro Appassionato, Paris, Belfond.

WIPPLINGER, Jonathan (2007). «The Aural Shock of Modernity: Weimar's Experience of Jazz», The Germanic Review, vol. 82, n 4, p. 299-320. 